\title{
BMJ Open Protocol and statistical analysis plan for the PREventing cardiovascular collaPse with Administration of fluid REsuscitation during Induction and Intubation (PREPARE II) randomised clinical trial
}

Derek W Russell (D) , ${ }^{1,2}$ Jonathan D Casey, ${ }^{3}$ Kevin W Gibbs, ${ }^{4}$ James M Dargin, ${ }^{5}$ Derek J Vonderhaar, ${ }^{6}$ A M Joffe, ${ }^{7}$ Shekhar Ghamande, ${ }^{8}$ Akram Khan, ${ }^{9}$ Simanta Dutta, ${ }^{4}$ Janna S Landsperger, ${ }^{3}$ Sarah W Robison, ${ }^{1}$ Itay Bentov, ${ }^{7}$ Joanne M Wozniak, ${ }^{5}$ Susan Stempek, ${ }^{5}$ Heath D White, ${ }^{8}$ Olivia F Krol, ${ }^{9}$ Matthew E Prekker, ${ }^{10,11}$ Brian E Driver, ${ }^{10}$ Joseph M Brewer, ${ }^{12}$ Li Wang, ${ }^{3}$ Christopher John Lindsell, ${ }^{3}$ Wesley H Self, ${ }^{13}$ Todd W Rice, ${ }^{3}$ Matthew W Semler, ${ }^{3}$ David Janz, ${ }^{14}$ PREPARE II Investigators

To cite: Russell DW, Casey JD, Gibbs KW, et al. Protocol and statistical analysis plan for the PREventing cardiovascular collaPse with Administration of fluid REsuscitation during Induction and Intubation (PREPARE II) randomised clinical trial. BMJ Open 2020;10:e036671. doi:10.1136/ bmjopen-2019-036671

- Prepublication history and additional material for this paper are available online. To view these files, please visit the journal online (http://dx.doi. org/10.1136/bmjopen-2019036671).

DWR and JDC contributed equally.

MWS and DJ contributed equally.

Received 07 January 2020 Revised 26 May 2020 Accepted 01 June 2020

Check for updates

(C) Author(s) (or their employer(s)) 2020. Re-use permitted under CC BY-NC. No commercial re-use. See rights and permissions. Published by BMJ.

For numbered affiliations see end of article.

Correspondence to Dr Derek W Russell; dwrussell@uabmc.edu

\section{ABSTRACT}

Introduction Cardiovascular collapse is a common complication during tracheal intubation of critically ill adults. Whether administration of an intravenous fluid bolus prevents cardiovascular collapse during tracheal intubation remains uncertain. A prior randomised trial found fluid bolus administration to be ineffective overall but suggested potential benefit for patients receiving positive pressure ventilation during tracheal intubation. Methods and analysis The PREventing cardiovascular collaPse with Administration of fluid REsuscitation during Induction and Intubation (PREPARE II) trial is a prospective, multi-centre, non-blinded randomised trial being conducted in 13 academic intensive care units in the USA. The trial will randomise 1065 critically ill adults undergoing tracheal intubation with planned use of positive pressure ventilation (non-invasive ventilation or bag-mask ventilation) between induction and laryngoscopy to receive $500 \mathrm{~mL}$ of intravenous crystalloid or no intravenous fluid bolus. The primary outcome is cardiovascular collapse, defined as any of: systolic blood pressure $<65 \mathrm{~mm} \mathrm{Hg}$, new or increased vasopressor administration between induction and 2 min after intubation, or cardiac arrest or death between induction and 1 hour after intubation. The primary analysis will be an unadjusted, intention-to-treat comparison of the primary outcome between patients randomised to fluid bolus administration and patients randomised to no fluid bolus administration using a $\chi^{2}$ test. The sole secondary outcome is 28-day in-hospital mortality. Enrolment began on 1 February 2019 and is expected to conclude in June 2020.

Ethics and dissemination The trial was approved by either the central institutional review board at Vanderbilt University Medical Center or the local institutional review board at each trial site. Results will be submitted for

\section{Strengths and limitations of this study}

- This multi-centre, randomised, controlled trial with target enrolment of 1065 patients will provide the highest quality available evidence for an important question in a commonly encountered clinical scenario.

- Broad eligibility criteria and enrolment at multiple centres will increase the external validity of the findings.

- Blinding is impractical due to the nature of this study intervention.

- The trial is not designed to examine the effects of fluid composition or volume of fluid administered.

publication in a peer-reviewed journal and presented at scientific conferences.

Trial registration number NCT03787732.

\section{INTRODUCTION}

Tracheal intubation is common in the care of critically ill patients but is associated with a high incidence of complications. ${ }^{1-3}$ Cardiovascular collapse is a composite of lifethreatening haemodynamic complications of tracheal intubation comprised of postintubation hypotension, ${ }^{4-6}$ administration of vasopressors to treat hypotension, cardiac arrest and death. Cardiovascular collapse occurs in $20 \%-30 \%$ of critically ill patients undergoing tracheal intubation, ${ }^{78}$ and is associated with increased in-hospital mortality. ${ }^{569}$ 
Some airway management experts recommend the intravenous administration of a fluid bolus beginning prior to induction (ie, the administration of procedural drugs such as anaesthetics) to prevent cardiovascular collapse during tracheal intubation. ${ }^{40}$ A fluid bolus could address the haemodynamic perturbations induced by induction and tracheal intubation, which include vasodilatory effects of induction medications, increased venous capacitance due to decreased circulating catecholamines and decreased venous return secondary to positive pressure applied to the thoracic cavity. However, the only reported trial to examine administration of a pre-intubation fluid bolus, the PrePARE (Preventing cardiovascular collaPse with Administration of fluid Resuscitation before Endotracheal intubation) trial, reported that a pre-intubation fluid bolus had no effect on the overall rate of cardiovascular collapse. ${ }^{8}$ The receipt of positive pressure ventilation, however, appeared to modify the effect of a fluid bolus administration on cardiovascular collapse in the PrePARE trial. Patients receiving positive pressure ventilation appeared to have a lower rate cardiovascular collapse in the fluid bolus group compared with the no fluid bolus group, both among patients receiving non-invasive ventilation for pre-oxygenation (RR $0.51 ; 95 \%$ CI 0.24 to 1.09 ; $\mathrm{p}$ value for interaction $=0.032$ ) and among patients receiving bag-mask ventilation between induction and laryngoscopy (RR $0.61 ; 95 \%$ CI 0.33 to 1.13 ; p value for interaction $=0.008) .{ }^{8}$

Provision of positive pressure ventilation with a bagmask device between induction and laryngoscopy has been shown to decrease the incidence of severe hypoxaemia during tracheal intubation of intensive care unit (ICU) patients (relative risk, 0.48 ; $95 \%$ CI 0.30 to 0.77 ). ${ }^{11}$ These results, and others examining use of non-invasive ventilation for pre-oxygenation during ICU intubations, ${ }^{12}$ suggest that positive pressure ventilation should be provided during tracheal intubation for most critically ill patients. ${ }^{10}$ This increases the importance of investigating the finding from the PrePARE trial that a pre-induction fluid bolus might prevent cardiovascular collapse among patients receiving positive pressure ventilation. We designed the PREventing cardiovascular collaPse with Administration of fluid REsuscitation during Induction and Intubation (PREPARE II) trial to examine the hypothesis that administration of a fluid bolus beginning prior to induction will decrease the incidence of cardiovascular collapse among critically ill adults undergoing tracheal intubation with positive pressure ventilation between induction and laryngoscopy.

\section{METHODS AND ANALYSIS}

This manuscript was written in accordance with Standard Protocol Items: Recommendations for Interventional Trials (SPIRIT) guidelines (see table 1 and online supplementary file 1 , section 1$).{ }^{13}$

\section{Patient and public involvement}

Materials used to communicate about the study with patients and family members were developed with input from the Vanderbilt Community Advisory Council. Study authors will disseminate the results of this study online and via social media in forms suitable for public understanding.

\section{Study design}

The PREPARE II trial is a pragmatic, multi-centre, un-blinded, parallel group, randomised trial. Among critically ill adults undergoing tracheal intubation undergoing positive pressure ventilation between induction and laryngoscopy, PREPARE II compares incidence of cardiovascular collapse between patients administered intravenous administration of a $500 \mathrm{~mL}$ fluid bolus and those receiving no fluid bolus administration. The trial protocol was approved with waiver of informed consent by either the central institutional review board at Vanderbilt University Medical Center or the local institutional review board at each trial site. An independent data and safety monitoring board (DSMB) is monitoring the progress and safety of the trial.

\section{Study sites}

PREPARE II is being conducted in 13 ICUs at academic medical centres across the USA. Site characteristics are listed in online supplementary file 2 , section 2 .

\section{Population}

In order to maximise the generalisability of this trial, the target population is meant to be broad and encompass all patients in whom the treating clinician judges there to be clinical equipoise on the use of the intervention. The trial includes adults (age $\geq 18$ years) located in a participating ICU for whom the treating clinicians have determined that tracheal intubation is required and for whom the planned procedural approach includes an operator who routinely performs tracheal intubation in the participating unit, administration of sedation (with or without neuromuscular blockade) and positive-pressure ventilation between induction and laryngoscopy. The trial excludes pregnant women and prisoners. In order for clinicians to not feel compelled to provide or withhold an intervention which they feel is wrong for a given patient, the trial also excludes patients for whom the treating clinicians feel (based on their clinical judgement at the time of enrolment) that the urgency of the intubation precludes safe performance of study procedures or that fluid bolus administration is either required or contraindicated.

\section{Randomisation and treatment allocation}

Patients are randomised in a 1:1 ratio to intravenous fluid bolus administration or no fluid bolus administration in permuted blocks of two, four or six, stratified according to study site. Study-group assignments (see online supplementary file 1, section 3; figure S1) are placed in sequentially numbered opaque envelopes and remain concealed until after enrolment. After enrolment and randomisation, patients, treating clinicians and study personnel are not blinded to study group assignment. 
Table 1 Standard Protocol Items: Recommendations for Interventional Trials checklist

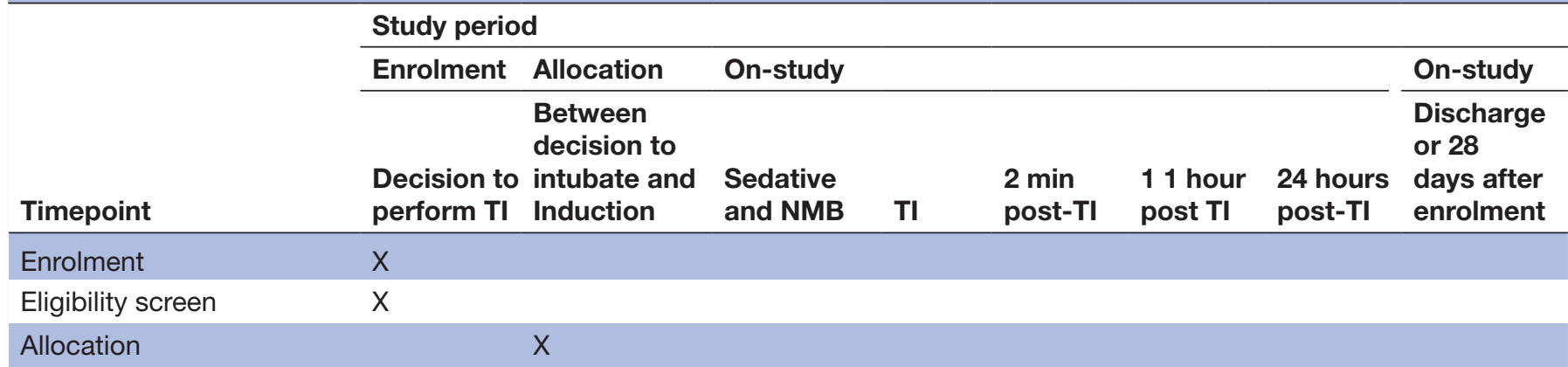

Interventions

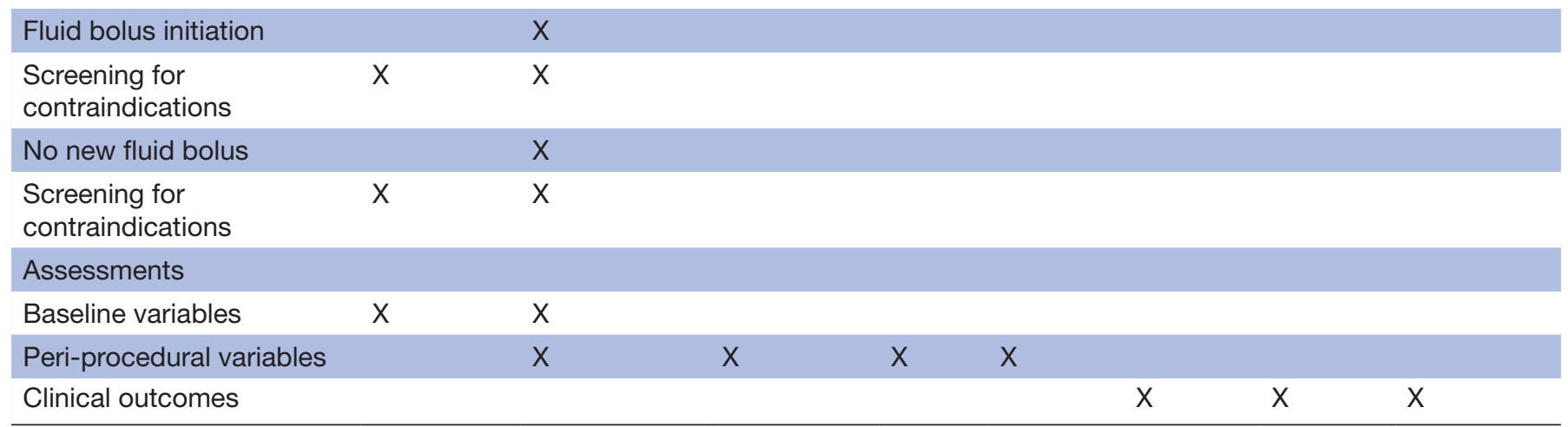

Baseline variables obtained from medical record include: demographic characteristics, Acute Physiology and Chronic Health Evaluation II (APACHE II) score and presence of sepsis/septic shock. Peri-procedural data collected by independent, trained observer includes the following: whether fluids were infusing prior to enrolment, receipt of the study intervention, the volume of study crystalloid infused (induction and 2 min after procedure), use of prophylactic vasopressor (or prophylactically increased vasopressor dose), addition of new vasopressor (or increased vasopressor dose) and systolic blood pressure (at baseline and nadir from induction to 2 min after procedure). Peri-procedural data collected by operator includes: sedation drugs used (and doses), oxygenation/ventilation modality between induction and laryngoscopy, and procedural complications. Clinical outcomes include: vital status (overall in-hospital death, cardiac arrest death within 1 hour of TI), number of ventilator-free days to 28 days, and number of intensive care unit-free days to 28 days.

NMB, neuromuscular blockade; TI, tracheal intubation.

\section{Study interventions}

Fluid bolus group

For patients who are assigned to the fluid bolus group, intravenous infusion of $500 \mathrm{~mL}$ of a crystalloid solution of the operator's choosing is initiated after randomisation and prior to induction. The fluid bolus is infused from above the level of the intravenous or intra-osseous access and allowed to infuse by gravity, manual pressure or bag pressure. The fluid bolus is discontinued after $500 \mathrm{~mL}$ have infused. For patients assigned to the fluids bolus group who are already receiving a fluid infusion, administration of $500 \mathrm{~mL}$ of fluids between randomisation and induction is achieved with either an additional bolus or increasing the rate of the existing infusion.

No fluid bolus group

For patients who are assigned to the no fluid bolus group, intravenous fluid administration is not initiated between randomisation and induction. Intravenous fluid infusions initiated prior to randomisation are not altered.

Cointerventions

Regardless of study group assignment, treating clinicians determine the timing of induction and tracheal intubation. Treating clinicians may stop infusion of a fluid bolus, increase or decrease the rate of infusion, or add a new fluid bolus at any time if felt to be required for the optimal care of the patient. Study group assignment determines only the initiation of intravenous fluid bolus administered between randomisation and induction. Figure 1 depicts the timeline of study procedures in the context of the tracheal intubation procedure.

Because the study enrols only patients for whom treating clinicians plan to administer positive-pressure ventilation between induction and laryngoscopy, most patients receive either non-invasive ventilation or bag-mask ventilation between induction and laryngoscopy. Instances in which positive-pressure ventilation between induction and laryngoscopy is not administered are recorded, along with the reason that positive-pressure ventilation was not administered (eg, emesis arising between randomisation and induction).

Treating clinicians determine the decision to intubate, modality and timing of pre-oxygenation, choice, dose and timing of medications for induction and neuromuscular blockade, decision to administer vasopressors before or after induction, choice of laryngoscope, use of cricoid 


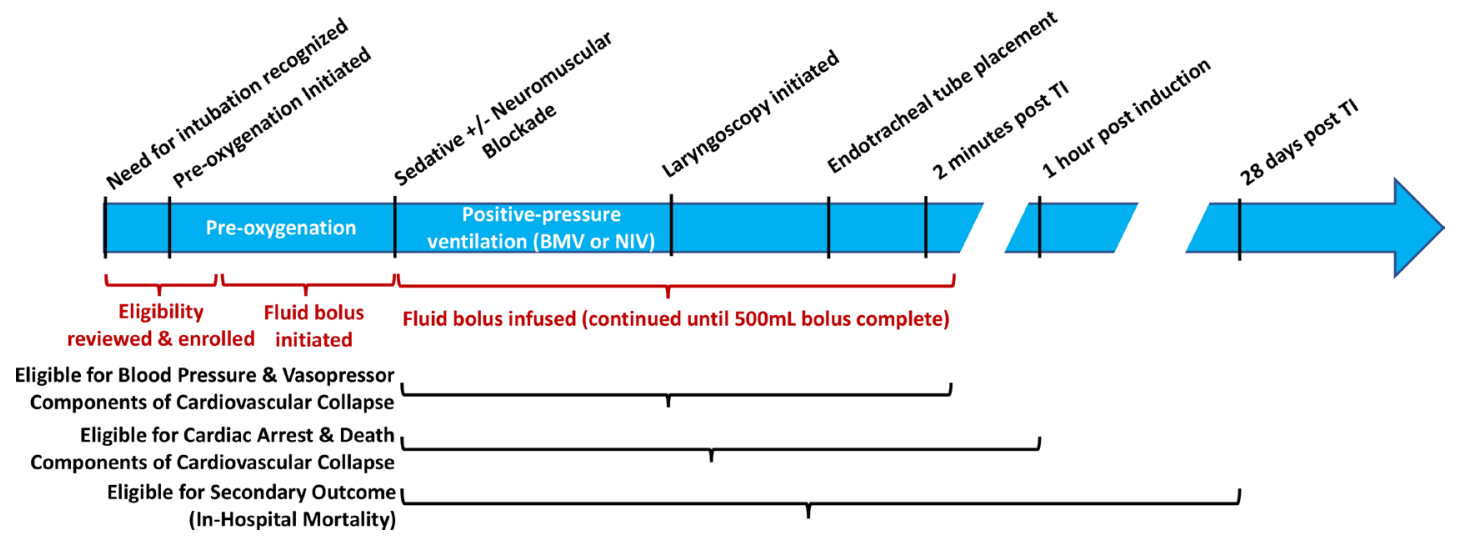

Figure 1 Timeline of tracheal intubation (TI), enrolment, study interventions and primary/secondary outcome eligibility in an enrolled patient.

pressure, method of positive pressure ventilation (noninvasive ventilation or bag-mask ventilation) between induction and laryngoscopy, decision to administer intravenous fluid for the treatment of hypotension, and use of additional airway management equipment and personnel. Data on these cointerventions are prospectively collected.

In some participating units, patients may be coenrolled in a randomised trial comparing use of Bougie or stylet in patients undergoing intubation emergently (BOUGIE) versus use of an endotracheal tube with stylet on the first attempt at tracheal intubation (ClinicalTrials.gov, NCT03928925). An interaction between the interventions evaluated in these trials in not anticipated and the results will be reported separately.

\section{Data collection}

Data collection for this study is described in detail in online supplementary file 1 , section 4 and table 1 provides further detail on data collection procedures.

\section{Primary outcome}

The primary outcome is cardiovascular collapse, defined as the occurrence of one or more of the following: systolic blood pressure $(\mathrm{SBP})<65 \mathrm{~mm} \mathrm{Hg}$ between induction and 2 min after intubation; new or increased vasopressor administration between induction and 2 min after intubation; cardiac arrest between induction and 1 hour after intubation; or death between induction and 1 hour after intubation.

Cardiovascular collapse is a commonly used endpoint in airway management research. ${ }^{48}$ Cardiovascular collapse is considered a 'reasonably likely surrogate endpoint' for short-term mortality because a strong mechanistic rationale links severe hypotension and cardiac arrest to short-term mortality and interventions that prevent cardiovascular collapse might reasonably be expected to prevent short-term mortality. ${ }^{14}$ Cardiovascular collapse was the primary outcome of the recently completed PrePARE trial, ${ }^{8}$ on which the design of the PREPARE II trial was based. In the PrePARE trial, the absolute risk of in-hospital mortality was $16.7 \%$ (95\% CI $3.4 \%$ to $30.0 \%$ ) higher among patients who experienced cardiovascular collapse during intubation compared with patients who did not. ${ }^{8}$

\section{Secondary outcome}

The sole secondary outcome is 28-day all-cause in-hospital mortality (online supplementary file 1, section 5). Short-term mortality is a commonly used patient-centred clinical endpoint for randomised trials in intensive care medicine and may be mechanistically associated with the primary outcome of cardiovascular collapse.

\section{Exploratory clinical outcomes}

- Each individual component of the composite primary endpoint:

- $\mathrm{SBP}<65 \mathrm{~mm} \mathrm{Hg}$ between induction and $2 \mathrm{~min}$ after intubation.

- New or increased vasopressor administration between induction and 2 min after intubation.

- Cardiac arrest between induction and 1 hour after intubation.

- Death between induction and 1 hour after intubation.

- Lowest SBP between induction and 2 min after intubation.

- Change in SBP from induction to lowest SBP between induction and $2 \mathrm{~min}$ after intubation.

- Ventilator-free days to 28 days (defined in online supplementary file 1 , section 6 ).

- ICU-free days to 28 days (defined in online supplementary file 1 , section 7 ).

\section{Exploratory safety outcomes}

- Lowest arterial oxygen saturation between induction and 2 min after intubation.

- Incidence of hypoxaemia (oxygen saturation <90\%) between induction and 2 min after intubation.

- Incidence of severe hypoxaemia (oxygen saturation $<80 \%$ ) between induction and 2 min after intubation.

- Oxygen saturation at 24 hours after intubation.

- Fraction of inspired oxygen at 24 hours after intubation. 
- Positive end expiratory pressure at 24 hours after intubation.

- SBP at 24 hours after intubation.

\section{Exploratory process measures}

- Initiation of an intravenous fluid bolus between induction and 2 min after intubation.

- Time from induction to successful intubation.

- Incidence of successful intubation on the first laryngoscopy attempt.

- Number of laryngoscopy attempts.

- Cormack-Lehane grade of glottic view on first attempt.

- Operator-assessed difficulty of intubation.

- Need for additional airway equipment or a second operator.

\section{Initial sample size estimation}

In a prior randomised trial comparing fluid bolus administration beginning prior to induction versus no fluid bolus administration in the same setting as the current trial, the incidence of cardiovascular collapse was 19.6\% in the fluid bolus group and $18.3 \%$ in the no fluid bolus group overall. However, among the subgroup of patients assigned to receive positive pressure ventilation with a bag-mask device between induction and laryngoscopy, the incidence of cardiovascular collapse was $16.0 \%$ in the fluid bolus group and $26.2 \%$ in the no fluid bolus group (10\% absolute risk difference and $40 \%$ relative risk difference). Assuming more conservative rates of cardiovascular collapse of $16.25 \%$ in the fluid bolus group and $25.0 \%$ in the no fluid bolus group $8.75 \%$ absolute risk difference and $35 \%$ relative risk difference), we calculated that enrolling 714 patients would provide $80 \%$ statistical power at a two-sided alpha level of 0.05 . Anticipating less than $5 \%$ missing data for the primary outcome, the initial planned enrolment for the trial was 750 patients. The study protocol included a pre-specified sample size re-estimation following the single interim analysis (see the Sample size re-estimation section).

\section{DSMB and interim analysis}

A DSMB composed of experts in clinical trials, critical care medicine, anaesthesia and emergency medicine is overseeing the design and conduct of the trial. The DSMB conducted a single interim analysis for efficacy and safety at the anticipated halfway point of the trial, after enrolment of 375 patients, on 12 November 2019. Stopping criteria were pre-specified in the study protocol, suggesting termination of the trial at the interim if the $p$ value for the difference between groups in the incidence of the primary outcome (cardiovascular collapse) or secondary outcome (28-day in-hospital mortality) were 0.001 or less using a $\chi^{2}$ test. Using this conservative Haybittle-Peto boundary $(\mathrm{p} \leq 0.001)$ allows the final analysis at the end of the trial to be performed using an unchanged level of significance.

The DSMB also formally evaluated the trial for safety and examined the highest fraction of inspired oxygen, highest positive end expiratory pressure and lowest arterial oxygen saturation at 24 hours after intubation in each study group. The prespecified early stopping criteria for physiological outcomes were as follows: if the $p$ value for the difference between study groups in any of these three physiological variables were 0.001 or less using a MannWhitney rank-sum test and concordant in direction with the point-estimate for mortality.

At the interim analysis, finding that no stopping criteria had been met and no safety concerns were observed, the DSMB recommended continuing the trial.

\section{Sample size re-estimation}

The study protocol specified that, after completion of the interim analysis and the recommendation to continue enrolment, 'the DSMB will evaluate the rate of the primary outcome in the no fluid bolus group. If the incidence of the primary outcome in the no fluid bolus group differs from the original estimate of $25.0 \%$, the DSMB may ask that the investigators perform a sample size re-estimation to maintain adequate statistical power to detect the planned relative risk difference in the primary outcome between groups'.

After completion of the interim analysis and the recommendation to continue enrolment, the DSMB examined the number of patients that would need to be enrolled in order to maintain $80 \%$ statistical power to detect the planned relative risk reduction of $35 \%$ in the primary outcome. Based on this information, the DSMB recommended increasing the total sample size from 750 to 1065 patients. The investigators accepted the DSMB's recommendation, revising the planned sample size for the final trial to 1065 patients. During the sample size re-estimation, both the study investigators and the DSMB remained blind to all outcomes by study group. No further interim analyses are planned.

\section{Statistical analysis principles}

R V.3.5.2 (R Foundation for Statistical Computing, Vienna, Austria) will be used for all analyses. Continuous variables will be reported as mean $\pm \mathrm{SD}$ or median and IQR; categorical variables will be reported as frequencies and proportions. Between-group comparisons will be made with the Mann-Whitney rank-sum test for continuous variables, and the $\chi^{2}$ test for categorical variables.

\section{Primary analysis of the primary outcome}

The primary analysis will be an unadjusted, intention-totreat comparison of patients randomised to the fluid bolus group versus patients randomised to the no fluid bolus group with regard to the primary outcome of cardiovascular collapse. Between group differences will be tested using an unadjusted $\chi^{2}$ test. A p value $<0.05$ will be used to indicate statistical significance for the primary analysis.

\section{Secondary analyses of the primary outcome}

To account for potential confounders, we will develop a logistic regression model with cardiovascular collapse (primary outcome) as the dependent variable and 
independent variables to include study group (fluid bolus group vs no fluid bolus group) and relevant confounders (age, Acute Physiology and Chronic Health Evaluation II (APACHE II) score at enrolment, presence of sepsis or septic shock, vasopressor receipt in the hour prior to enrolment, and receipt of intravenous fluid infusion initiated prior to enrolment). We will also develop a logistic regression model accounting for the above variables plus any baseline characteristics that appear on visual review to be potentially imbalanced between the study groups.

Because patients within a specific ICU may be more similar to other patients within the same ICU than to patients in other ICUs, we will fit a generalised linear mixed-effects model with the outcome of cardiovascular collapse, including group assignment as a fixed effect and study unit (stratification variable) as a random effect.

We will repeat the primarily analysis using alternative definitions of cardiovascular collapse, including: (1) using an SBP $<90 \mathrm{~mm} \mathrm{Hg}$ rather than an $\mathrm{SBP}<65 \mathrm{~mm}$ $\mathrm{Hg}$, (2) using 28-day in-hospital mortality rather than death within 1 hour and (3) using days from enrolment to in-hospital death (defined in online supplementary file 1 , section 8) rather than death within 1 hour.

Interpreting composite endpoints can be challenging when the components have different levels of clinical importance. We will repeat the primary analysis of the primary outcome using a global rank scale. Use of a hierarchical global rank score places greater weight on the objective, patient-centred clinical outcomes (death, cardiac arrest) than on the immediate physiological outcomes (hypotension and vasopressors). The global rank endpoint will be constructed by comparing each patient with every other patient in the study and assigning a score for each pairwise comparison based on whom fared better. To make the pairwise comparison, we will consider a priority order of endpoints: (1) death within 1 hour of intubation; (2) cardiac arrest within 1 hour of intubation; (3) SBP $<65 \mathrm{~mm} \mathrm{Hg}$ between induction and 2 min after intubation; and (4) new or increased vasopressor administration between induction and $2 \mathrm{~min}$ after intubation. The scores will be summarised and compared between study groups (fluid bolus group vs no fluid bolus group) using an unadjusted Mann-Whitney U test.

Given the findings of the PrePARE trial subgroup analysis (ie, that the effect of fluid bolus administration on cardiovascular collapse may be related to the receipt of positive pressure ventilation during intubation), ${ }^{8}$ we will repeat the primary analysis excluding patients who did not receive positive pressure during intubation. Because many critical care patients are already receiving intravenous fluid for other indications when the decision is made to intubate and this may modify the effect of a new fluid bolus, we will repeat the primary analysis excluding patients who were already receiving intravenous fluid at the time of enrolment.

\section{Analysis of effect modification for the primary outcome}

We will examine whether pre-specified baseline variables modify the effect of treatment group on the primary outcome using formal tests of statistical interaction in a logistic regression model. Independent variables will include study group assignment, the potential effect modifier of interest, and the interaction between the two (eg, study group xpresence of sepsis or septic shock). Significance will be determined by the $\mathrm{p}$ value for the interaction term, with values less than 0.10 considered to suggest of a potential interaction and values less than 0.05 considered to confirm an interaction. Continuous variables will be analysed using restricted cubic splines and preferentially displayed as continuous variables with 3-5 knots using a locally weighted regression or partial effects plots. We will use a forest plot to display the effect of covariates. If required for data presentation, continuous variables will be dichotomised for inclusion in a forest plot. We will examine whether the following baseline variables modify the effect of study group on the primary outcome:

- APACHE II score at enrolment (continuous variable).

- Presence of sepsis or septic shock at time of enrolment (yes/no).

- Receipt of vasopressors in the 1 hour prior to enrolment (yes/no).

- Predicted probability of cardiovascular collapse as calculated by a pre-specified multivariable model (continuous variable).

In addition to the above variables which can be assessed prior to study enrolment, we will perform exploratory analyses examining additional potential effect modifiers that are intended to represent patient physiology at baseline, but which are collected between enrolment and induction and therefore have the theoretical potential to be affected by study group assignment. These include:

- Receipt of positive pressure ventilation for preoxygenation (via either non-invasive mechanical ventilation or bag-mask ventilation) (yes/no).

- Choice of sedative medication (etomidate, ketamine, propofol, other).

- New or increased vasopressor administration prior to or with induction (yes/no).

- SBP at induction (continuous variable in $\mathrm{mm} \mathrm{Hg}$ ).

- Oxygen saturation at induction (continuous variable in \%).

Finally, to examine our assumption that no interaction will exist between the interventions evaluated in the PREPARE II and BOUGIE trials, among patients coenrolled to these trials, we will examine whether BOUGIE group assignment modifies the primary outcome. If, contrary to our expectation, an interaction is confirmed (based on criteria listed above for interaction testing), the BOUGIE group assignment will be added to the adjustment model for the primary outcome of cardiovascular collapse. 


\section{Analysis of the secondary outcome}

The sole secondary outcome of 28-day in-hospital mortality will be compared between patients randomised to the fluid bolus group vs patients randomised to the no fluid bolus group using an unadjusted $\chi^{2}$ test.

\section{Analyses of exploratory outcomes}

All pre-specified exploratory outcomes will be compared between patients randomised to the fluid bolus group versus patients randomised to the no fluid bolus group. Continuous outcomes will be compared with the MannWhitney $\mathrm{U}$ test and categorical variables with the $\chi^{2}$ test. In a sensitivity analysis using data only from each patient's first tracheal intubation in the PREPARE II dataset, we will compare the fluid group to the no fluid bolus group with regard to in-hospital mortality, ventilator-free days and ICU-free days.

\section{Handling of missing data}

Although we have allowed for up to $5 \%$ missingness in our power calculation, we do not anticipate that data for the primary outcome of cardiovascular collapse will be missing for any patients. Missing data will not be imputed for the primary or secondary outcome. In adjusted analyses, missing data for covariates may be imputed using a multiple imputation technique.

\section{Corrections for multiple testing}

We pre-specify a single primary analysis of a single primary outcome, and a single secondary analysis with one outcome. All additional analyses are deemed hypothesisgenerating, and no corrections for multiple comparisons will be performed.

\section{Trial status}

The PREPARE II trial is a pragmatic, prospective, multicentre, non-blinded randomised clinical trial comparing fluid bolus to no fluid bolus during tracheal intubation of critically ill adults. Patient enrolment began on 1 February 2019 and is expected to be completed in June 2020.

\section{ETHICS AND DISSEMINATION \\ Informed consent}

In current clinical practice, initiating an intravenous fluid bolus beginning prior to tracheal intubation and not administering an intravenous fluid bolus beginning prior to tracheal intubation are both common management approaches, with significant variation between providers. ${ }^{15}$ All patients eligible for this trial would have either received or not received an intravenous fluid bolus for tracheal intubation as a part of their clinical care, regardless of participation in the trial. To be eligible for the trial, patients' treating clinicians must feel that initiation of a new fluid bolus for tracheal intubation is neither required nor contraindicated for the patient's optimal care. The protocol states that a fluid bolus can be given or withheld for patient safety at any time in the study, regardless of group assignment. For these reasons, the trial is felt to pose minimal incremental risk compared with the clinical care patients would receive outside of the trial.

Tracheal intubation of critically ill adults is commonly an urgent or emergent procedure for which obtaining informed consent for the clinical procedure or informed consent for research is impracticable.

This information was provided to either the central institutional review board at Vanderbilt University Medical Center or the local institutional review board at each trial site (see online supplemental file 1, section 2), and the trial was approved with a waiver of informed consent.

\section{Information for patients and families}

Information regarding the study is made available to patients and families through three mechanisms: (1) a patient and family notification sheet provided to each patient and family following enrolment informing the patient of his or her enrolment and describing the study, (2) a patient and family information sheet containing general information about the study and contact information for the research team displayed in at least three publicly visible locations within the study unit, (3) a patient and family information sheet containing general study information and contact information for the research team provided to each patient and family at the time of admission to the study unit. The mechanism(s) of providing information to patients and families used by each study site was determined by local site investigators and local IRBs and is described in online supplementary file 1, section 2; table S1.

\section{Protocol changes}

Any changes to the trial protocol will be recorded on ClinicalTrials.Gov as per SPIRIT guidelines (see online supplementary file 1 , section 9 ).

\section{Data handling}

For details of privacy and data handling, see online supplementary file 1 , section 10 .

\section{Dissemination plan}

Trial results will be submitted to a peer-reviewed journal for consideration of publication and will be presented at scientific conferences.

\section{Author affiliations}

${ }^{1}$ Department of Medicine, Division of Pulmonary, Allergy, \& Critical Care Medicine, University of Alabama at Birmingham, Birmingham, Alabama, USA

${ }^{2}$ Veterans Integrated Service Network 7, Department of Veterans Affairs, Washington, District of Columbia, USA

${ }^{3}$ Department of Medicine, Division of Allergy, Pulmonary and Critical Care Medicine, Vanderbilt University School of Medicine, Nashville, Tennessee, USA

${ }^{4}$ Department of Medicine, Section of Pulmonary, Critical Care, Allergy and Immunologic Disease, Wake Forest School of Medicine, Winston-Salem, North Carolina, USA

${ }^{5}$ Department of Medicine, Division of Pulmonary and Critical Care Medicine, Lahey Hospital and Medical Center, Burlington, Massachusetts, USA

${ }^{6}$ Department of Pulmonary and Critical Care Medicine, Ochsner Health System, New Orleans, Louisiana, USA

${ }^{7}$ Department of Anesthesiology and Pain Medicine, University of Washington, Seattle, Washington, USA 
${ }^{8}$ Department of Medicine, Division of Pulmonary Disease and Critical Care Medicine, Baylor Scott \& White Medical Center, Temple, Texas, USA

${ }^{9}$ Department of Medicine, Division of Pulmonary and Critical Care Medicine, Oregon Health \& Science University School of Medicine, Portland, Oregon, USA

${ }^{10}$ Department of Emergency Medicine, Hennepin County Medical Center, Minneapolis, Minnesota, USA

${ }^{11}$ Department of Medicine, Division of Pulmonary/Critical Care Medicine, Hennepin County Medical Center, Minneapolis, Minnesota, USA

${ }^{12}$ Department of Medicine, Division of Pulmonary, Critical Care, and Sleep Medicine, University of Mississippi Medical Center, Jackson, Mississippi, USA

${ }^{13}$ Department of Emergency Medicine, Vanderbilt University School of Medicine,

Nashville, Tennessee, USA

${ }^{14}$ Department of Medicine, Section of Pulmonary/Critical Care Medicine and Allergy/ Immunology, Louisiana State University School of Medicine in New Orleans, New Orleans, Louisiana, USA

Collaborators List of PREPARE II Investigators: Wake Forest School of Medicine - Kevin W. Gibbs, MD ${ }^{\star \star *}$; Simanta Dutta, MD ${ }^{\star \star *}$; Stephen P Peters MD, PhD*; Muhammad Ali, MBBS*; Rita N. Bakhru, MD, MS*; Scott Bauer, ANP-BC*; Christina R. Bellinger, MD*; Amanda M. Brown, PA-C*; Blair Brown, MD*; Jerri Brown, ADN, RN*; Caitlin Bumgarner, ACGNP*; Wendy Butcher, RN, BSN*; Megan Caudle, ACGNP*; Arjun B. Chatterjee, MD*; David J. Chodos, MD*; Gerardo Corcino, RN,

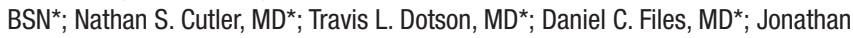
L Forbes, DO*; John P. Gaillard, MD*; Katherine A. Gershner, D0*; Shannon Ginty, PA-C*; Kiadrick R. Hood, RN, MSN, CMSRN*; April Hazelwood, ADN, RN*; Katherine Hendricks, FNP*; Kelly Jacobus, PA-C*; Jonathan T. Jaffe, MD*; Stacy Kay, ACGNP*; Chad A. Kloefkorn, MD*; Jennifer Krall, MD*; Margo T. Lannan, MD*; Cornelia Lane, ACGNP*; Cynthia Lanning, BSN, RN*; Jessica Lyons, PA-C*; William I. Mariencheck Jr., MD*; Chad R. Marion, DO, PhD*; Matthew A. Maslonka, MD*; Sara McClintock, ACGNP*; Nathaniel M. Meier, MD*; Matthew C. Miles, MD, MEd*; Peter J. Miller, MD*; Sophia Mitchell, PA- $C^{*}$; Wendy C. Moore, MD*; Katherine Moss, PA-C*; Andrew M. Namen, MD*; Dustin L. Norton, MD*; Stella B. Ogake, MD*; Jill A. Ohar MD*; Victor E. Ortega, MD, PhD*; Jessica A. Palakshappa, MD, MS*; Rodolfo M. Pascual, MD*; Sandi Pascual, ANP-BC*; Aaron Pickens, MD*; Adam R Schertz, MD*; Matt Strong, ADN, RN*; Alexander 0. Sy, MD*; Braghadheeswar Thyagarajan MD*; Amy Townsend, ACGNP*; Russell Worthen, FNP-BC*; Michael Wlodarski, PA-C*; Charles Yarbrough, ADN, RN*; Caroline York, PA-C*; Vanderbilt University Medical Center-Jonathan D. Casey, MD, MSc ${ }^{\star \star *}$; Janna S. Landsperger, MSN" ${ }^{\star \star *} ; \mathrm{Li}$ Wang, MS ${ }^{\star * *}$; Christopher J. Lindsell PhD ${ }^{\star * *}$; Todd W. Rice, MD, MSc ${ }^{* * *}$; Matthew W. Semler, MD, MSc ${ }^{\star \star *}$; Wesley H. Self, MD, MPH ${ }^{\star \star *}$; Bradley Lloyd, RRT-ACCS*; Lahey Hospital and Medical Center - James Dargin, MD ${ }^{\star \star *}$; Joanne Wozniak, PA-C***; Susan Stempek, PA-C***; Christopher Adler, PA-C*; Ahmed Agameya, $\mathrm{MD}^{*}$; Michael Colancecco, DO*; Daniel Fitelson, MD*; Joshua Giaccotto, MD*; Gena Han, DO*; Louise Kane, MD*; Ezra Miller, MD*; Timothy Noland, PA-C*; Jaqueline Price, PA-C*; Joseph Plourde, PA-C*; Emily Adams, PA-C*; Fraser Mackay, MD*; Laura Mahoney, PA-C*; Avignat Patel, MD*; Michael Plourde, PA-C*; Zena Saadeh, PA-C*; Sara Shadchehr, DO*; Sandeep Somalaraju, MD*; Eleanor Summerhill, MD*; Ryan Webster, MD*; Jordan Winnicki, PA-C*; Ekaterina Yavarovich, DO*; University

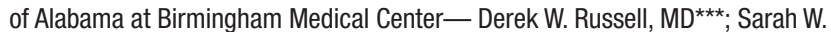
Robison, MD**; Sheetal Gandotra, MD**; Swati Gulati, MBBS, MS ${ }^{\star *}$; Anna AltzStamm RN, BSN, CCRN; Cristina Bardita, MD, PhD* ; Mary Clay Boone RN, BSN*; Joe W. Chiles III, MD*; Kristina Collins RN, BSN; Abby Drescher RN, BSN*; Kevin G. Dsouza, MD*; Janna Dunn, RN, AND*; Stacy Ejem, MD*; Josh Gautney, MD*; Nicole Harris, RN, ADN*; Savannah Herder, RN, BSN*; Tamer Hudali, MD, MPH*; R. Chad Wade, MD*; Rutwij Joshi, MBBS*; Daniel Kelmenson, MD*; Anne Merrill Mason RN, $\mathrm{BSN}^{*}$; Scott R. Merriman, MD*; Takudzwa Mkorombindo, MD*; Megan Moore, RN,

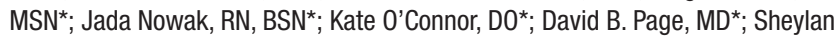
D. Patel, MD*; G. Bruno Pereira, MD, PhD*; Lisa Sarratt RN, BSN*; Tabitha Stewart RN, BSN*; William S. Stigler, MD*; Kadambari Vijaykumar, MBBS*; Gina White RN,

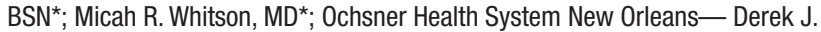
Vonderhaar, MD ${ }^{\star \star *}$; Kevin M. Dischert MD ${ }^{\star *}$; University of Washington Harborview Medical Center-Aaron M. Joffe, D0**; Itay Bentov, MD, PhD ${ }^{* * *}$; Christopher Barnes $^{\star *}$; Andrew M. Walters ${ }^{\star *}$; Baylor Scott \& White Medical Center- Shekhar Ghamande, MD**; Heath D. White, D0, MS**; Alejandro C. Arroliga, MD, MS ${ }^{\star *}$; Tasnim Lat, D0**; Oregon Health and Science University School of MedicineAkram Khan, MD**; Olivia F. Krol, BS ${ }^{\star \star *}$; Stephanie Nonas, MD ${ }^{\star *}$; Milad K. Jouzestani**; Raya Adi*; Chandani Anandkat*; Hanae Benchbani, MD*; Matthew G. Drake, MD*; Makrina N. Kamel, BS*; Ramanpreet Randhawa, MD*; Jessica L. Tsui, MD*; Hennepin County Medical Center— Matthew E. Prekker, MD**; Brian E. Driver, MD**; University of Mississippi Medical Center- Joseph M. Brewer, DO ${ }^{\star \star *}$; Louisiana State University School of Medicine—David R. Janz, MD, MSc.

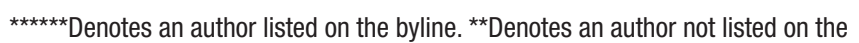
byline due to space considerations. *Denotes a collaborator.

Contributors All study authors approved the final version of this manuscript. Study concept and design: DWR, JDC, MWS, DJ, CJL, LW, WHS and TWR; acquisition of data: DWR, JDC, KWG, JMD, DJV, AMJ, SG, AK, SD, JSL, SWR, IB, JMW, HDW, OFK, MEP, BED, JMB, KWG, SG, AK, WHS, SS, TWR, MWS. DJ and the PREPARE II investigators; drafting of the manuscript: DWR, JDC, DJ, MWS; critical revision of the manuscript for important intellectual content: DWR, JDC, KWG, JMD, DJV, AMJ, SG, AK, SD, JSL, SWR, IB, JMW, HDW, OFK, MEP, BED, JMB, WHS, SS, CJL, LW, TWR, MWS, DJ and the PREPARE II investigators; study supervision: DJ and MWS.

Funding JDC was supported in part by the NHLBI (2T32HL087738-12 and K12HL133117). MWS was supported in part by the NHLBI (K23HL143053). DWR was supported in part by the UAB Walter Frommeyer, Jr. Fellowship in Investigative Medicine and by the Department of Veteran's Affairs (VISN 7 Research Development Award). Data collection used the Research Electronic Data Capture (REDCap) tool developed and maintained with Vanderbilt Institute for Clinical and Translational Research grant support (UL1 TR002234 from NCATS/NIH). The funding institutions had no role in (1) conception, design, or conduct of the study, (2) collection, management, analysis, interpretation or presentation of the data or (3) preparation, review or approval of the manuscript.

Competing interests None declared.

Patient consent for publication Not required.

Provenance and peer review Not commissioned; externally peer reviewed.

Supplemental material This content has been supplied by the author(s). It has not been vetted by BMJ Publishing Group Limited (BMJ) and may not have been peer-reviewed. Any opinions or recommendations discussed are solely those of the author(s) and are not endorsed by BMJ. BMJ disclaims all liability and responsibility arising from any reliance placed on the content. Where the content includes any translated material, BMJ does not warrant the accuracy and reliability of the translations (including but not limited to local regulations, clinical guidelines, terminology, drug names and drug dosages), and is not responsible for any error and/or omissions arising from translation and adaptation or otherwise.

Open access This is an open access article distributed in accordance with the Creative Commons Attribution Non Commercial (CC BY-NC 4.0) license, which permits others to distribute, remix, adapt, build upon this work non-commercially, and license their derivative works on different terms, provided the original work is properly cited, appropriate credit is given, any changes made indicated, and the use is non-commercial. See: http://creativecommons.org/licenses/by-nc/4.0/.

ORCID iD

Derek W Russell http://orcid.org/0000-0002-2716-1344

\section{REFERENCES}

1 Simpson GD, Ross MJ, McKeown DW, et al. Tracheal intubation in the critically ill: a multi-centre national study of practice and complications. Br J Anaesth 2012;108:792-9.

2 Martin LD, Mhyre JM, Shanks AM, et al. 3,423 emergency tracheal Intubations at a university hospital: airway outcomes and complications. Anesthesiology 2011;114:42-8.

3 Leibowitz AB. Tracheal intubation in the intensive care unit: extremely hazardous even in the best of hands. Crit Care Med 2006;34:2497-8.

4 Jaber S, Jung B, Corne P, et al. An intervention to decrease complications related to endotracheal intubation in the intensive care unit: a prospective, multiple-center study. Intensive Care Med 2010;36:248-55.

5 Green RS, Turgeon AF, Mclntyre LA, et al. Postintubation hypotension in intensive care unit patients: a multicenter cohort study. J Crit Care 2015;30:1055-60.

6 Ferrada P, Manzano-Nunez R, Lopez-Castilla V, et al. Meta-Analysis of post-intubation hypotension: a plea to consider circulation first in hypovolemic patients. Am Surg 2019;85:167-72.

7 Perbet S, De Jong A, Delmas J, et al. Incidence of and risk factors for severe cardiovascular collapse after endotracheal intubation in the ICU: a multicenter observational study. Crit Care 2015;19:257.

8 Janz DR, Casey JD, Semler MW, et al. Effect of a fluid bolus on cardiovascular collapse among critically ill adults undergoing tracheal intubation (prepare): a randomised controlled trial. Lancet Respir Med 2019;7:1039-47.

9 Heffner AC, Swords DS, Nussbaum ML, et al. Predictors of the complication of postintubation hypotension during emergency airway management. J Crit Care 2012;27:587-93. 
10 Higgs A, McGrath BA, Goddard C, et al. Guidelines for the management of tracheal intubation in critically ill adults. $\mathrm{Br} J$ Anaesth 2018;120:323-52.

11 Casey JD, Janz DR, Russell DW, et al. Bag-Mask ventilation during tracheal intubation of critically ill adults. $N$ Engl J Med 2019;380:811-21.

12 Baillard C, Prat G, Jung B, et al. Effect of preoxygenation using noninvasive ventilation before intubation on subsequent organ failures in hypoxaemic patients: a randomised clinical trial. Br J Anaesth 2018;120:361-7.
13 Chan A-W, Tetzlaff JM, Gøtzsche PC, et al. Spirit 2013 explanation and elaboration: guidance for protocols of clinical trials. BMJ 2013;346:e7586.

14 Cagney DN, Sul J, Huang RY, et al. The FDA NIH biomarkers, endpoints, and other tools (best) resource in neuro-oncology. Neuro Oncol 2018;20:1162-72.

15 Green RS, Fergusson DA, Turgeon AF, et al. Resuscitation prior to emergency endotracheal intubation: results of a national survey. West J Emerg Med 2016;17:542-8. 